\title{
The Correlation Between the Gravitational Constant and Distance from Matter
}

\author{
Abolfazl Soltani \\ Department of Physics, University of Birjand, Birjand, Iran \\ Email: soltani.a.phyics@gmail.com
}

\begin{abstract}
Here I show that we are able to consider a large value for the gravitational constant, $G$, at the vicinity of matter $(r<1 \AA)$. Today physics and chemistry extremely need to this choice. The large value of $G$ causes significant results in physics and chemistry. By considering a large value for $G$, at the vicinity of matter, the stability of atom and atomic and molecular bonds can be explained by a different method. Moreover, here I prove that the value of vacuum permittivity, $\varepsilon_{0}$, at the vicinity of a charged particle $(r<1 \AA)$, is larger than its present value and the Spacetime lines interact with electric field lines. Also, I obtain a new equation (not a new value) for a very important constant, namely fine-structure constant, with the presence of the gravitational constant, $G$.
\end{abstract}

Keywords: Gravitational Constant, Vacuum Permittivity, Fine-Structure Constant, Spacetime, Electric Field

Introduction: There is gravitational force at the subatomic scale $(r<1 \AA)$ but its value is very negligible. By considering a large value for $G$, at the subatomic scale, the force of gravity can be increased at this scale. But the presence of a strong gravitational force along with the Coulomb force cause the collapse of the Bohr atom. To avoid this problem, we must choose a different value for $\varepsilon_{0}$, at the subatomic scale. In the following, we will discuss about these issues and show that selecting a large value for $G$ at the atomic scale has very valuable results.

\section{Laboratory Limitations}

First we should say that there is no laboratory limitations for selecting a large value for $G$ at atomic distances because the value of $G$ at the vicinity $(r<1 \AA$ or $r<1 \mathrm{~nm})$ of Protons, Neutrons, Electrons and nuclei of atoms has not been measured yet. The basis of most of the experiments to measure the value of $G$ is the Newton's law of universal gravitation $\left(F=G m_{1} m_{2} / r^{2}\right)$ and torsion Balance [1]. In these experiments [2-5] the value of $G$ is obtained according to the Newton's law and the values of $m_{1}, m_{2}, F$ and $r$. In addition, the other various experiments with different methods have been carried out to measure the value of $G$ such as the ones using very cold atoms and atom interferometry [6-9]. Yet, none of these experiments measured the value of $G$ at distances of less than one nanometer or one angstrom $(r<1 \AA$ or $r<1 \mathrm{~nm})$. Therefore, we do not know the value of $G$ through experiments, at subatomic distances; and thus we can theoretically consider a large value for it. We have: 


$$
\begin{cases}G(r)=G_{0}=6.67 \times 10^{-11} \mathrm{Nm}^{2} \mathrm{Kg}^{-2} & r>1 \AA \\ G(r)=G_{1} \gg G_{0} & r<1 \AA\end{cases}
$$

In the above equations, the boundary between $G_{0}$ and $G_{1}$ (namely $r=1 \AA$ ) is an approximate and selective one, with no emphasis on it.

\section{Physical Concept of Change in the Gravitational Constant}

The presence of an object in space causes the curvature of Spacetime (Fig. 1). The passing of gravitational waves creates the wrinkles in Spacetime [10,11], and variation in the value of $G(r)$ from $G_{0}$ to $G_{1}$ can be illustrated as the convergence and compression of Spacetime lines. It means that in Fig. 1, the Spacetime lines, at the vicinity of matter $(r<1 \AA)$, converge and the squares become smaller.

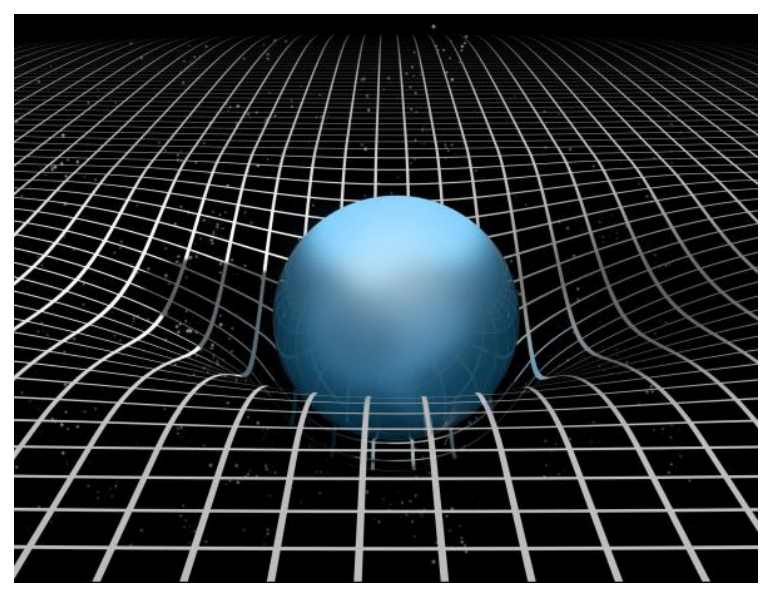

Fig. 1. The presence of an object in space causes the curvature of Spacetime. The Spacetime lines are shown in this figure.

\section{Stability of Atom and Gravitational Effects}

By considering a large value for $G$ at the subatomic scale, the gravitational effect of the nucleus along with the coulomb force must be considered as the reason for the stability of the electrons around the nucleus of atom. Based on this, in this section, we will investigate the simplest atom, namely the hydrogen atom. By using the Bohr atomic model we will obtain new equations for the radius of the hydrogen atom $\left({ }_{1}^{1} H\right)$, its mechanical energy, Rydberg constant and fine-structure constant. First, we need to review the Bohr atomic model.

In the Bohr model, Bohr assumed that the electron revolves in a circular orbit about the nucleus. and he supposed that the mass of the electron is completely negligible compared to the mass of the nucleus [12] The potential energy for the hydrogen atom is coulomb potential [12][13][14]:

$$
E_{P}(r)=-\int F(r) d r=-\frac{k_{e} e^{2}}{r}
$$

And the centripetal force is: 


$$
\frac{k_{e} e^{2}}{r^{2}}=\frac{m_{e} v^{2}}{r} \Rightarrow v=\sqrt{\frac{k_{e} e^{2}}{m_{e} r}}
$$

64 Where $k_{e}$ is coulomb constant. In the Bohr model, the weak gravitational potential is ignored, so the mechanical energy of the system is [13][14]:

6

$$
E=E_{K}+E_{P}=\frac{1}{2} m_{e} v^{2}+\left(-\frac{k_{e} e^{2}}{r}\right) \stackrel{\frac{k_{e} e^{2}}{r^{2}}=\frac{m v^{2}}{r}}{\rightleftharpoons} E=-\frac{k_{e} e^{2}}{2 r}
$$

In Bohr model the orbital angular momentum of the electron, $L=m_{e} v r$, must be a constant, because the force acting on the electron is entirely in the radial direction. Applying the Bohr quantization condition to $L$ [13], we have

$$
L=n \hbar \Rightarrow m_{e} v r=n \hbar
$$

Therefore, we will obtain the following equations for atomic radius [13][14]:

$$
\begin{gathered}
m_{e} v r=n \hbar \stackrel{v=\sqrt{\frac{k_{e} e^{2}}{m_{e} r}}}{\Longrightarrow} r_{n}=\frac{n^{2} \hbar^{2}}{k_{e} m_{e} e^{2}} \quad n=1,2,3, \ldots . \\
r_{1}=\frac{\hbar^{2}}{k_{e} m_{e} e^{2}}=0.528 \AA
\end{gathered}
$$

And

$$
r_{n}=n^{2} r_{1}
$$

Where $n$ is the Principal Quantum number. For orbital velocity of electron, we have:

$$
\begin{gathered}
v=\frac{n \hbar}{m_{e} r} \stackrel{(5)}{\Rightarrow} v_{n}=\frac{k_{e} e^{2}}{n \hbar} \\
v_{1}=\frac{k_{e} e^{2}}{\hbar}
\end{gathered}
$$

As you know, the definition of fine-structure constant or Somerfield's constant is the ratio of the speed of electron in the first orbit of Bohr atom to the velocity of light in vacuum [14]:

$$
\alpha=\frac{v_{1}}{c} \stackrel{(9)}{\Rightarrow} \alpha=\frac{k_{e} e^{2}}{\hbar c}=\frac{1}{137}
$$

In addition, by using the equation 3 we have:

$$
\begin{gathered}
E_{n}=-\frac{k_{e} e^{2}}{2 r_{n}}=-\frac{k_{e} e^{2}}{2 n^{2} r_{1}} \stackrel{(6)}{\Rightarrow} E_{n}=-\frac{k_{e}^{2} e^{4} m_{e}}{2 n^{2} \hbar^{2}} \\
E_{1}=-\frac{k_{e}^{2} e^{4} m_{e}}{2 \hbar^{2}}=-13.5 \mathrm{eV}
\end{gathered}
$$




$$
E_{n}=-\frac{E_{1}}{n^{2}}
$$

Based on Bohr model, the difference between the two energy states in the hydrogen atom is equal to $h v$ :

$$
v=\frac{E_{u p}-E_{\text {low }}}{h} \stackrel{(13)}{\Longrightarrow} v=\frac{E_{1}}{h}\left(\frac{1}{n_{l}^{2}}-\frac{1}{n_{u}^{2}}\right) \stackrel{c=v \lambda}{\Longrightarrow} \frac{1}{\lambda}=\frac{E_{1}}{h c}\left(\frac{1}{n_{l}^{2}}-\frac{1}{n_{u}^{2}}\right)
$$

Where $n_{u}$ and $n_{l}$ are the quantum numbers for the upper and lower energy states, respectively; and $\lambda$ is the wavelength of radiation emitted by the atom[13][14]. The equation 14 is of precisely the same mathematical form as the empirically derived Rydberg formula:

$$
\frac{1}{\lambda}=R\left(\frac{1}{n_{l}^{2}}-\frac{1}{n_{u}^{2}}\right)
$$

Therefore:

$$
R=\frac{E_{1}}{h c} \stackrel{(12)}{\Longrightarrow} R=\frac{k_{e}^{2} e^{4} m_{e}}{4 \pi \hbar^{3} c}=1.0974 \times 10^{-3} \AA^{-1}
$$

Compare this equation with the experimentally determined value $1.0968 \times 10^{-3} \AA^{-1}$. This experimental value is determined by the precise Spectroscopic Methods [14]. The strong agreement of the experimental value and the theoretical value of Rydberg constant was a great success for the Bohr model.

Now, we want to do recent calculations in the presence of the powerful gravitational effects of the nucleus of the hydrogen atom. Since the orbital velocity of the electron is much lower than the velocity of light $\left(v_{1} / c=1 / 137\right)$ [12], the gravitational potential $(\mathrm{gP})$ of the hydrogen atom $\left({ }_{1}^{1} H\right)$ is non-relativistic, namely

$$
E_{g P}(r)=-\frac{G_{1} m_{e} m_{p}}{r} \quad r<1 \AA
$$

Where $m_{e}$ and $m_{p}$ are the mass of electron and proton, respectively. In such a case, the new potential energy $\left(E_{P n e w}\right)$ is equal to the sum of gravitational potential $\left(E_{g P}\right)$ and electrical potential $\left(E_{e P}\right)$. The mechanical energy is:

$$
E=E_{K}+E_{\text {Pnew }}=E_{K}+\left[E_{g P}+E_{e P}\right]
$$

It is clear, in such a case, the values of $m_{e}, m_{p}$ and $G_{1}$ will enter in the calculations and the recent equations such as equation 6,10 and 16 will change; therefore, the theoretical and experimental results will not be equal. To avoid this problem, new potential energy $\left(E_{\text {Pnew }}\right)$ must be equal to old potential energy (equation 2). It means that, we will have a new value for Coulomb constant (namely $k_{\text {enew }}$ instead of $k_{e}$ ), at the vicinity of electric charge $(r<1 \AA$ ). So we have:

$$
E_{\text {Pold }}=E_{\text {Pnew }} \Rightarrow-\frac{k_{e} e^{2}}{r}=-\frac{k_{\text {enew }} e^{2}}{r}-\frac{G_{1} m_{e} m_{p}}{r} \Rightarrow k_{\text {enew }}=k_{e}-\frac{G_{1} m_{e} m_{p}}{e^{2}}
$$


114 This is a very exciting idea. But, what does it mean? As you know, the definition of Coulomb 115 constant is $1 / 4 \pi \varepsilon_{0}$. Therefore, the variation of Coulomb constant from $k_{e}$ to $k_{\text {enew }}$ means that 116 when we approach to an electric charge, the amount of vacuum permittivity shifts from $\varepsilon_{0}$ to $117 \varepsilon_{0 \text { new }}$. This is because of the variation of the gravity constant from $G_{0}$ to $G_{1}$ and the convergence 118 and compression of Spacetime lines. Interestingly, because of the compression of Spacetime lines 119 near the charged particle the vacuum permittivity changes. It means that the Spacetime lines 120 interact with electric field lines.

121 So, based on equation 18, we have:

$$
k_{\text {enew }}<k_{e} \stackrel{k_{e}=\frac{1}{4 \pi \varepsilon_{0}}}{=} \varepsilon_{0 \text { new }}>\varepsilon_{0}
$$

123

35

In the presence of gravity, the mechanical energy of the hydrogen atom is equal to

$$
E=E_{K}+E_{P}=\frac{1}{2} m_{e} v^{2}+\left(-\frac{k_{\text {enew }} e^{2}}{r}-\frac{G_{1} m_{e} m_{p}}{r}\right)
$$

And the centripetal force is:

$$
\begin{aligned}
& \frac{m_{e} v^{2}}{r}=\frac{k_{\text {enew }} e^{2}}{r^{2}}+\frac{G_{1} m_{e} m_{p}}{r^{2}} \\
& v=\sqrt{\frac{k_{\text {enew }} e^{2}+G_{1} m_{e} m_{p}}{m_{e} r}}
\end{aligned}
$$

By putting the equation 22 in equation 20, we have:

$$
E=\frac{-\left(k_{\text {enew }} e^{2}+G_{1} m_{e} m_{p}\right)}{2 r}
$$

Compare this equation with equation 3 . For atomic radius we have:

$$
\begin{gathered}
m_{e} v r_{n}=n \hbar \Rightarrow r_{n}=\frac{n \hbar}{m_{e} v} \stackrel{(22)}{\Longrightarrow} r_{n}=\frac{n^{2} \hbar^{2}}{m_{e}\left(k_{\text {enew }} e^{2}+G_{1} m_{e} m_{p}\right)} \quad n=1,2,3, \ldots \\
r_{1}=\frac{\hbar^{2}}{m_{e}\left(k_{\text {enew }} e^{2}+G_{1} m_{e} m_{p}\right)}
\end{gathered}
$$

$$
\text { (23) } \stackrel{(24)}{\Longrightarrow} E_{n}=\frac{-m_{e}\left(k_{\text {enew }} e^{2}+G_{1} m_{e} m_{p}\right)^{2}}{2 n^{2} \hbar^{2}}
$$


137

138

139

140

141

142

143

144

145

146

147

148

149

150

151

152

153

154

155

156

157

158

159

160

161

162

163

164

Where $R_{\text {new }}$ is the new equation of Rydberg constant with the presence of $G_{1}, m_{e}$ and $m_{p}$. Compare equations 28 and 29 with equation 16. This new value of Rydberg constant is equal to its recent value $\left(1.0974 \times 10^{-3} \AA^{-1}\right)$ because of using equation 18 . Addition to equation 22 the orbital velocity of electron is:

$$
m_{e} v r=n \hbar \stackrel{(24)}{\Longrightarrow} v_{n}=\frac{\left(k_{\text {enew }} e^{2}+G_{1} m_{e} m_{p}\right)}{n \hbar}
$$

And

$$
\alpha_{\text {new }}=\frac{v_{1}}{c}=\frac{\left(k_{\text {enew }} e^{2}+G_{1} m_{e} m_{p}\right)}{c \hbar}
$$

This is the new equation of the fine-structure constant. Compare this equation with equation 10. According to equation 18 , the value of $\alpha_{\text {new }}$ is the same value as $\alpha(1 / 137)$. In the new equation of the fine-structure constant, $\alpha_{\text {new }}$, special Relativity (c), electromagnetism $\left(k_{\text {enew }}\right)$, Quantum mechanics ( $\hbar$ ) and General relativity $\left(G_{1}\right.$ and $m$ ) are unified by the number $1 / 137$.

Eventually, based on all of these discussions, the Coulomb force between the two charged particles like two electrons is equal to

$$
\begin{array}{ll}
F=\frac{k_{e} e^{2}}{r^{2}}=\frac{1}{4 \pi \varepsilon_{0}} \frac{e^{2}}{r^{2}} & r>1 \AA \\
F=\frac{k_{\text {enew }} e^{2}}{r^{2}}=\frac{1}{4 \pi \varepsilon_{\text {onew }}} \frac{e^{2}}{r^{2}} & r<1 \AA
\end{array}
$$

\section{Atomic and Molecular Bonds}

In this article, we show that a large value for $G$ can be considered at the atomic scale. Based on this, in this section, we will investigate how the hydrogen molecule is composed of two Hydrogen atoms. A Hydrogen molecule is formed of the collision of two Hydrogen atoms in the laboratory and inside a star. Consider two hydrogen atoms $\left({ }_{1}^{1} H\right)$ collide with each other. Because of the strong gravitational force between the two electrons $\left(F=G_{1} m_{e}^{2} / r^{2}\right.$ ), the electrons of the two hydrogen atoms will be attracted to each other (Fig. 2). In such a case, the distance ( $r$ ) between the two electrons gradually narrows; and the Coulomb force $\left(F=k_{\text {enew }} e^{2} / r^{2}\right)$ gradually increases. Thus, the two electrons will approach together until the attraction gravitational force equals the repulsion Coulomb force. In this way, the bond between the two atoms of hydrogen is formed and the hydrogen molecule is formed. Here the nuclei of the two hydrogen atoms also interact with each other and the electrons. But, the electron-electron interaction is the most effective interaction in the hydrogen molecule. 


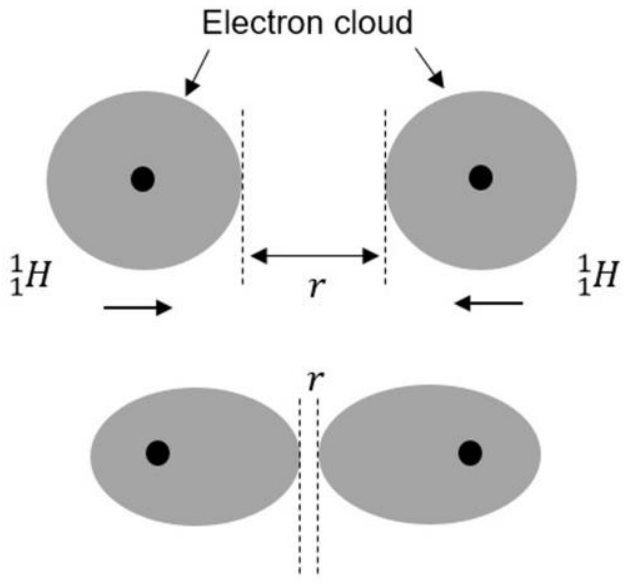

Hydrogen Molecule

Quantum shape
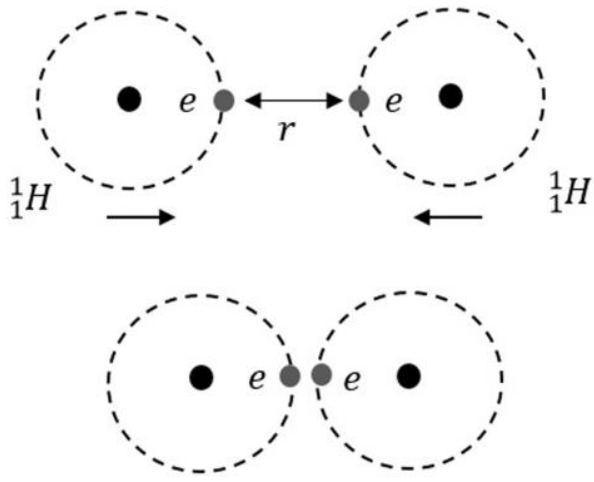

Hydrogen Molecule

Classical shape
165

166

167

168

169

170

171

172

173

174

175

176

177

178

179

180

181

182

183

184

185

186

187

188

189

Fig. 2. Hydrogen molecule formation. The two hydrogen atoms are attracted and form a bond. In the hydrogen molecule, the repulsion Coulomb force between the two electrons is equal to the attraction Gravitational force, based on this article. In the hydrogen molecule and in Quantum shape, the electron clouds of two atoms do not overlap because of the repulsion Coulomb force between the two electrons.

By using this description about the hydrogen molecule formation, it is easy to explain other atomic and molecular bonds. In the bonds between atoms and molecules with multiple electrons, several attraction and repulsion forces (arising from the electrons of inner and outer shells of atoms and nuclei) are involved in the formation of bonds and the magnitude of Bond-dissociation energy. For example, in the $\mathrm{C}-\mathrm{H}$ bond, the electron of the hydrogen atom interacts with six electrons of Carbon atom and therefore, the bond energy of $\mathrm{C}-\mathrm{H}$ is different from $\mathrm{H}-\mathrm{H}$. For this reason, the energy required to break one bond is different from the other. Here and in atomic bonds and molecular bonds, the gravitational interactions and Coulomb interactions between the electron-electron, electron-nucleus and nucleus-nucleus, depending on the distance between the two particles, can be explained through $G_{1}$ and $K_{\text {enew }}$ or $G_{0}$ and $K_{e}$. In other words, in atomic bonds and molecular bonds, the particles which are close to each other $(r<1 \AA$ ), interact with each other with constants $G_{1}$ and $K_{\text {enew }}$ and the particles which are at a distance interacts with each other with constants $G_{0}$ and $K_{e}$.

\section{Conclusion}

In this article, we proved that considering a large value for $G$, at the vicinity of matter, is a very appropriate choice for physics and chemistry. The justification of atomic and molecular bonds is very acceptable and understandable than covalent bond [15], van der Waals bond [15] and some other bonds [15] in chemistry. This article revealed that Spacetime lines interact with electric field lines. This can be a way to know the nature of electric field, electric charge and also magnetic field. Moreover, in this article, we obtained new equations for Rydberg constant and fine-structure 
constant. The results and discussions of this paper will cause a great revolution in physics and chemistry.

\section{References:}

1. Quinn, T. Measuring big G. Nature 408, 919-921 (2000)

2. Cavendish, H. Experiments to determine the density of the Earth. Philosophical Transactions of the Royal Society of London 88, 469-526 (1798).

3. Gundlach, J. Markowitz, S. Measurement of Newton's Constant using a Torsion Balance with angular acceleration feedback. Phys. Rev. Lett. 85(14):2869-72 (November 2000).

4. Luo, J. Liu, Q. Tu, L. Determination of the Newtonian Gravitational Constant G with Time-ofSwing Method. Phys. Rev. Lett. 102, 240801 (16 June 2009).

5. Armstrong, T. Fitzgerald, M. New Measurements of G Using the Measurement Standards Laboratory Torsion Balance. Phys. Rev. Lett. 91, 201101 (13 November 2003).

6. Rosi, G. Sorrentino, F. Cacciapuoti, L. Prevedelli, M. \& Tino, G. Precision measurement of the Newtonian gravitational constant using cold atoms. Nature 510, 518-521 (26 June 2014).

7. Fixler, J. B. Foster, G. T. McGuirk, J. M. \& Kassevich, M. Atom Interferometer Measurement of the Newtonian Constant of Gravity. Science 315, 74-77 (2007)

8. Bertoldi, A. et al. Atom interferometry gravity-gradiometer for the determination of the Newtonian gravitational constant G. Eur. Phys. J. D 40, 271-279 (2006).

9. Lamporesi, G. Bertoldi, A. Cacciapuoti, L. Prevedelli, M. \& Tino, G. M. Determination of the Newtonian gravitational constant using atom interferometry. Phys. Rev. Lett. 100, 050801 (2008).

10. Ryder, L. Introduction to General Relativity (Cambridge University Press. Ed, 1. 2009)

11. Rindler, W. Essential Relativity: Special, General, and Cosmological. (Springer-verlag, ed. 2, 1977)

12. Bohr, N. On the Constitution of Atoms and Molecules. Philos. Mag. 26, 1, (1913)

13. Eisberg, R. Quantum Physics. (John Wiley \& Sons Inc, ed. 2, 1974), pp. 98-107

14. Weidner, R. \& Sells, R. Elementary Modern Physics (Allyn and Bacon, ed. 2, 1973), pp 206216

15. Levine, N. Quantum Chemistry (Prentice Hall, ed. 5, 1999) 